Review

\title{
Car-to-Pedestrian Communication Safety System Based on the Vehicular Ad-Hoc Network Environment: A Systematic Review
}

\author{
Peng Jing, Wei Huang * and Long Chen \\ School of Automobile and Traffic Engineering, Jiangsu University, Zhenjiang 212013, China; \\ jingpeng@ujs.edu.cn (P.J.); chenlong@ujs.edu.cn (L.C.) \\ * Correspondence: weihuang.ren@foxmail.com
}

Received: 3 August 2017; Accepted: 11 October 2017; Published: 14 October 2017

\begin{abstract}
With the unparalleled growth of motor vehicles, traffic accident between pedestrians and vehicles is one of the most serious issues in the word-wild. Plenty of injuries and fatalities are caused by the traffic accidents and crashes. The connected vehicular ad hoc network as an emerging approach which has the potential to reduce and even avoid accidents have been focused on by many researchers. A large number of car-to-pedestrian communication safety systems based on the vehicular ad hoc network are researching and developing. However, to our limited knowledge, a systematic review about the car-to-pedestrian communication safety system based on the vehicular ad-hoc network has not be written. The purpose and goal of this review is to systematically evaluate and access the reliability of car-to-pedestrian communication safety system based on the vehicular ad-hoc network environment and provide some recommendations for the future works according to throwing some light on the previous literatures. A quality evaluation was developed through established items and instruments tailored to this review. Future works are needed to focus on developing a valid as well as effective communication safety system based on the vehicular ad hoc network to protect the vulnerable road users.
\end{abstract}

Keywords: car-to-pedestrian communication; car-to-pedestrian safety; vehicular ad hoc networks (VANETs)

\section{Introduction}

An unparalleled revolution in the number of vehicles world-wide has been caused by the process of rapid urbanization and growth of the economy. The increase of vehicles in the last decade has result in a large number of crashes and put lots of pressures on the existing road [1]. Pedestrians who are one of the most vulnerable participants between pedestrians and vehicles collisions, leading to fatal injuries and even fatalities [2]. In the European Union, it is reported that over 300,000 vulnerable road users (VRUs, e.g., pedestrians and bicyclists) injuries and 4800 VRUs fatalities ever year, which account for roughly $16 \%$ of the total number of the injuries and $19 \%$ of the total number of fatalities [3]. In the United State, the 4743 pedestrian fatalities in 2012 represented an increase of 6 percent from 2011 and were the highest number of fatalities in the last 5 years. In 2012, pedestrian deaths accounted for 14 percent of all traffic fatalities, and made up 3 percent of all the people injured in traffic crashes [4]. According to the NHTSA (National Highway Traffic Safety Administration) report, a pedestrian was killed every two hours and injured every seven minutes in traffic crashes [5]. Furthermore, crashes between vehicles and pedestrians can also result in traffic congestions and economy cost [6]. This alarming increase in mortality and fatality has become a matter of great attention around the word.

Taking into account of all this, the safety between the pedestrians and vehicles have attracted great attention by researchers. Subsequently, two profoundly different research directions have been 
developed: pedestrian collision mitigation and collision avoidance. Collision mitigation attempts to alleviate the consequence caused by accidents [7]. Collision avoidance approaches refer to foreseeing and checking possible collision which makes vehicles stop before oncoming crashes [8].

In this review, the authors focus on pedestrian collision avoidance which has drawn significant attention in the last decade [9]. Conventional pedestrian protection systems can be based on two classes of pedestrian detection methods: (a) utilizing contact- or non-cooperative perception sensors to prevent and avoid pedestrian injure such as RADARs, LASER scanners, IR sensors and imaging sensors (computer vision) [10-16]. However, the limitation of the line-of-sight is a fundamental drawback of these technologies [2] (i.e., Pedestrians who are obstructed, e.g., Behind a parking truck or a building); (b) using wireless communication to transmit detected information among vehicles or between vehicles and pedestrians. Mizui, K.M. et al. [17-19] proposed the first modality of vehicular communication that used optical laser or infrared laser. Sam, D. et al. [20] pointed out the drawback of this system is that each vehicle can communicate with only two vehicles, which are the vehicle directly in front of it and the one directly behind it in the same lane. Another communication method was proposed by using Radio Frequency (RF) [21,22]. Here the vehicle can broadcast to all the vehicles in its range. Later in 1999, the Federal Communication Commissions (FCC) allocated a frequency spectrum for vehicle-to-vehicle and vehicle-to-roadside wireless communication, the commission then established Dedicated Short Range Communication (DSRC) service in 2003 [23]. As a result, in recent years, more attentions have been focused on integrating wireless communication with classic sensor-based methods in order to improve pedestrian road-safety $[24,25]$. The assigned frequency and newly generated services enable vehicles and roadside units to form vehicular Ad hoc Network (VANETs), where the nodes can communicate wirelessly with each other directly without central server [26]. Vehicles are becoming "computers on wheels" and the nodes of the networks [27].

Vehicular Ad Hoc Networks (VANETs) are emerging advanced technology integrating ad hoc network, wireless LAN and cellular technology to realize intelligent inter-vehicle communication and vehicle-to-roadside communication [24], which offered a potential platform between vehicles and infrastructures or among vehicles for exchanging information to improve the safety of the traffic situation. Lee, U. et al. [28] allowed some roadside nodes with wireless devices, such as pedestrians and other vulnerable road users joining vehicular communication for exchanging the information. Therefore, plenty of car-to-pedestrian communication safety systems based on the vehicular ad hoc network are emerging and designing. Sam, D. et al. [20] proposed a low cost reliable approach by using a hybrid VANET system that combines the advantages of both the VANET as well as the wireless sensor network. Combining the wireless roadside sensors and the VANET to detect the events on the road and to communicate with vehicles. Later, the author improved the system integrating the body sensors with the VANET [29], this system will give an alert to the driver ahead of time for avoiding the accident.

With the increase of electric devices especially smartphone, the role of distraction as a contributing factor to vehicle crash has gained heightened attention in recent years. Numerous studies have shown that driver attention is impaired by the use of mobile devices such as cell phone [30,31]. Recent studies have shown that pedestrian exhibit riskier road-crossing behavior when texting or talking on the cell phone [32-34]. The combination of drivers and pedestrians being distracted by mobile devices creates a particularly lethal mix. The source of the distraction (mobile technology) also offers a potential means of remediation to improve pedestrian safety. In recently, some scholars using the smart phone to replace the handhold device to comprise the vehicular ad hoc networks between vehicles and pedestrians without employing the roadside unit to communicate with each other. Qualcomm and Honda researchers have done to extend the DSRC technology by enabling DSRC in smart phones for communication between vehicles and pedestrian [35,36]. Sugimoto et al. [37] used the WLAN communication technology embedded in the smart phone to communicate with oncoming vehicles for avoiding traffic accident. Jose et al. [38] and Wu et al. [39] employed the Wi-Fi technology embedded in the phone to exchange the information between vehicles and pedestrians before collision. However, 
these proposed car-to-pedestrian communication safety systems have some limitations: (a) the short battery lives of the handhold devices or smart phones; (b) the data transmission quality was influenced by some factors (e.g., The obstacles; packets lost rate; transmission delay); (c) the accuracy or precision of the positioning device is not satisfied with the requirement of safety system; (d) these systems are developing and researching, which have not been applied in the real world by now. Although most of the systems have done the experiment to validate the reliability, the experiment done under certain specific condition which cannot be on behalf of the complex situations in real world. Owing to the flaws of these car-to-pedestrian systems, it is important to compare these systems and access the reliability of these systems for improving the safety of the traffic situation. Furthermore, the vehicular ad hoc network employed in the car-pedestrian safety is a newly and popular research field. A rigorous assessment of the existing literatures is essential because studies with poor designs, methodological flaws could result in unexpected results especially about the car-to-pedestrian safety.

In this review, authors are going to throw some light on the previous researches in this area and will access existing systems based on the vehicular ad hoc network by analyzing the car-pedestrian communication safety system reliability and seeking to advantages and limitations of these proposed communication safety systems. Eventually, according to the systematic evaluation and analysis, the authors propose some recommendations for the future works.

The remainder of the review is organized as follows: Section 2 describes the methods of selecting eligible papers. The systematic process of the selected review is presented in Section 3. Section 4 describes the quality of the reviewed studies. Limitations and strengths of this paper is proposed in Section 5. Section 6 provides the discussion. Conclusions are presented in Section 7. Eventually, the future works discusses in the last section.

\section{Method}

\subsection{Search Approach and Database Searched}

Five databases, Web of Science, ScienceDirect, Academic Search Complete, IEEE Xplore, TRID were searched using the advanced search by keywords contained in the title, abstract, and the main body of the papers according to the PRISMA [40] (preferred reporting items for systematic reviews and meta-analyses) statements. These databases are interdisciplinary, international large-scale comprehensive retrieval tools, which include engineering, statistics, computer science, and transportation et al. The searches included detailed search field related to vehicle-pedestrian safety, vehicle-pedestrian communication and vehicular ad hoc network. The three search fields were connected with "AND" to ensure at least one term of each field could be found in the results. Four groups of keywords were included and at least one category of keywords must be used in combination: (1) car/vehicle-pedestrian safety, car/vehicle-pedestrian accident, car/vehicle-pedestrian collision, car/vehicle-pedestrian crashes; (2) vehicle-to-vehicle communication, vehicle-to-pedestrian communication, vehicle-to- $\mathrm{X}$ communication, vehicle-to-infrastructure communication, V2P, V2V, V2X; (3) pedestrian safety, road users safety, vulnerable road users safety, vehicle safety, car safety; (4) connected vehicles, vehicular ad-hoc network , VANET, internet vehicles, cooperative vehicle. Using the advanced search in each of the five databases, the terms in each search field were link with "OR". The search must be conformed to match the database according to the database structure. It is vital for the author to identify the previous review. The references which contained in the identified papers were also reviewed for further studies.

\subsection{Inclusion and Exclusion Criteria}

To ensure the articles included in the review are eligible and valid, articles must satisfy the following criteria: (1) be published in English journal with a peer-reviewed and written in English; (2) including the car-pedestrian communication safety system; (3) the system using connected vehicle 
technology or ad-hoc network or VANET; (4) including the communication between vehicles and pedestrians to exchange the critical information.

\subsection{Data Extraction}

Using the matrix method extracted the standardized data extraction table. The information extracted from the each eligible and valid papers containing the data transmission assessment (e.g., packets lost rate, the distance of transmission, the speed of the vehicle, the speed of the pedestrian, the obstacles), the validation assessment (e.g., data, simulation, field experiment), and the accuracy of positioning assessment (e.g., the location of the vehicles, the location of the pedestrians). For the reliability of the extracted data, the authors drew out eight papers and extracted the information independently. $85 \%$ of the extracted information were approved by the authors, which indicating high inter-rater reliability.

\subsection{Quality Assessment}

In this review, the author using a modified checklist that contain the data transmission quality assessment and the validation quality assessment as well as the accuracy of the positioning assessment to carefully assess the quality of the eligible and valid papers.

The checklist include five factors to access the quality of the data transmission: packets lost rate, the distance of transmission, the speed of vehicles, the speed of pedestrians, and the obstacles on the road. Chika Sugimoto et al. [41] done experiment to analyze the relationship between the data transmission efficiency and the transmission distance. The transmission distance strongly influenced the transmission efficiency, therefore, the transmission distance become the assessment criterion. Packets lost rate is also an important factor affect the reliability of the communication safety system which is proposed by Liu et al. [42]. Thus, it is an indispensable element to assess the quality of the data transmission. Toru Hagiwara et al. [43] proposed that validation of the communication system should be under the dynamic conditions. Therefore, the speed between pedestrians and vehicles is selected to be one of criteria. In real road environment the data transmission changes greatly depending on the surroundings such as running and parked vehicles, buildings, trees, and traffic signals. Hence, the obstacle is another assessment criteria.

In order to evaluate the effects affecting the quality of validation, the modified checklist contains three factors: the data source, simulation and field experiment. The real data employed to simulate or do experiments can reflect the true existing problem of the road and surrounding environment. Assessment of different application and systems could be made via valid experiment but they are time costly and consume a large number of resources. Instead, Simulation can be employed to assess different simple or complicated or innovative solutions before implementation [44]. The field experiment can verify the model and the reliability of the communication safety system in the actual scenarios and environments.

The Table 1 list all the evaluated papers based on the following 9 items. The possible range of assessment scores as shown in Table 1.

Table 1. Checklist for evaluating studies' quality.

\begin{tabular}{ccc}
\hline Accessing Items & Description & Scores \\
\hline Accessing the Data Transmission Quality & \\
\hline \multirow{2}{*}{ The packets lost rate } & Include & 1 \\
& Not include & 0 \\
\hline \multirow{2}{*}{ The distance of transmission } & Include & 1 \\
& Not include & 0 \\
\hline
\end{tabular}


Table 1. Cont.

\begin{tabular}{|c|c|c|}
\hline Accessing Items & Description & Scores \\
\hline \multirow{2}{*}{ The obstacles } & Include & 1 \\
\hline & Not include & 0 \\
\hline \multirow{2}{*}{ The speed of vehicles } & Include & 1 \\
\hline & Not include & 0 \\
\hline \multirow{2}{*}{ The speed of pedestrians } & Include & 1 \\
\hline & Not include & 0 \\
\hline \multicolumn{3}{|c|}{ Assessing the Validation Quality } \\
\hline \multirow{2}{*}{ The data } & Experimental data & 1 \\
\hline & Real data & 2 \\
\hline \multirow{2}{*}{ Simulation } & Include & 1 \\
\hline & Not include & 0 \\
\hline \multirow{2}{*}{ Field experiment } & Include & 1 \\
\hline & Not include & 0 \\
\hline \multicolumn{3}{|c|}{ The Accuracy of the Positioning } \\
\hline \multirow{2}{*}{ The accuracy of the positioning device } & Include & 1 \\
\hline & Not include & 0 \\
\hline
\end{tabular}

\section{Systematic Review Process}

The process of searching and retrieving valid papers is shown in Figure 1. The count of papers extracted from each database were 4515 (Science Direct), 46 (Web of Science), 1559 (IEEE), 68 (Academic Search Complete), 60 (TRID), respectively. Removing duplicated papers, 4873 different records were collected from the five databases, after screening the titles and abstracts, 365 records were remaining and identifying. There are two reasons to exclude the invalid papers: (1) non-use the VANET communication technology; (2) non-involved car-pedestrian system. The reference lists of eliminating reviews were reviewed and potential papers were collected. Eventually, 20 available publications were left and satisfied the full criteria in this review as shown in Figure 1. The main details of 20 available works were demonstrated as shown in Table 2.

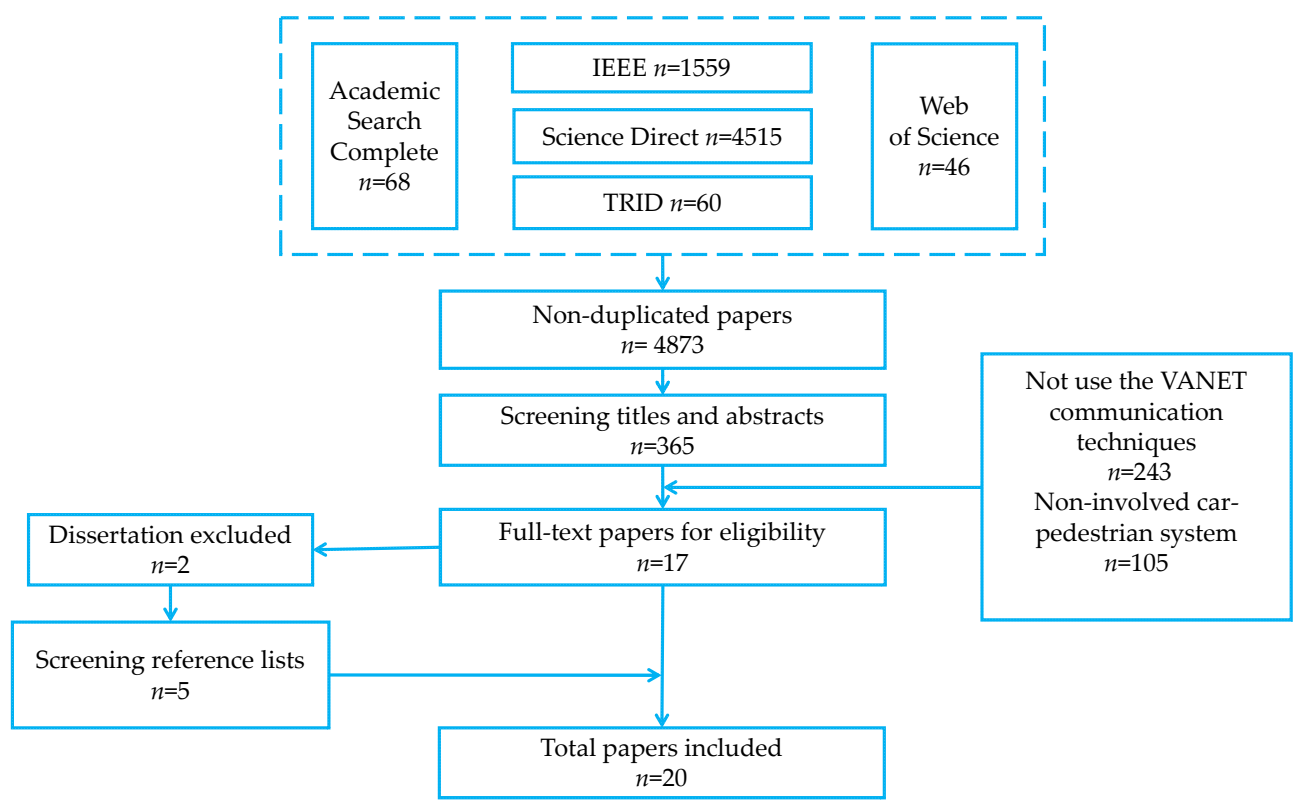

Figure 1. The flowchart of systematic review process. 
Table 2. Summary of the Selected Papers in this Review.

\begin{tabular}{|c|c|c|c|c|}
\hline Lead Author (Year) & Location & Journal & Components of the System & Objective \\
\hline $\begin{array}{l}\text { Hassan Artail } \\
\quad(2017)\end{array}$ & America & Wireless communication and moble comuting & $\begin{array}{l}\text { 1.LET network's Location server } \\
\text { 2.smartphone } \\
\text { 3.Road Side Units }\end{array}$ & $\begin{array}{l}\text { The main aim is to maximally avoid any road mortality } \\
\text { and improve the pedestrian safety. }\end{array}$ \\
\hline $\begin{array}{l}\text { Dahlia Sam } \\
\quad(2016)\end{array}$ & India & Vehicular Communication & $\begin{array}{l}\text { 1.Vehicle nodes } \\
\text { 2.Pedestrian body unit } \\
\text { 3.Road Side Unit } \\
\text { 4.Arduino microcontroller }\end{array}$ & $\begin{array}{l}\text { This system use the detected information to check the } \\
\text { chance of an accident occurring. }\end{array}$ \\
\hline $\begin{array}{l}\text { Zishan Liu } \\
\quad(2016)\end{array}$ & China & $\begin{array}{l}\text { International Journal of Distributed } \\
\text { Sensor Networks }\end{array}$ & $\begin{array}{l}\text { 1.The DSRC/LET/Wi-Fi OBU } \\
\text { 2.The tablet inside the vehicle } \\
\text { 3.Smart phone } \\
4 \text { The app server }\end{array}$ & $\begin{array}{l}\text { Evaluating the performance of the communication } \\
\text { system in terms of PDR and end-to-end latency. }\end{array}$ \\
\hline $\begin{array}{l}\text { Pierre Merdrignac } \\
\quad(2016)\end{array}$ & France & $\begin{array}{l}\text { IEEE Transactions on Intelligent } \\
\text { Transportation System }\end{array}$ & $\begin{array}{l}\text { 1.V2Pro Vu application } \\
\text { 2.Wi-Fi module } \\
\text { 3.laser sensors }\end{array}$ & Improving the safety of vnlunerable road users. \\
\hline $\begin{array}{l}\text { Ahmed Hussein } \\
\text { (2016) }\end{array}$ & Spain & Intelligent Transportation System & $\begin{array}{l}\text { 1.location sensors } \\
\text { 2.communciation module } \\
\text { 3.smartphone }\end{array}$ & $\begin{array}{l}\text { Reducing the risks related the use of mobile devices in a } \\
\text { traffic context and decresing the accident exposure of } \\
\text { pedestrians and other VRUs. }\end{array}$ \\
\hline $\begin{array}{l}\text { Liu Zhenyu } \\
\text { (2015) }\end{array}$ & China & $\begin{array}{l}\text { The journal of China University of Posts and } \\
\text { Telecommunications }\end{array}$ & $\begin{array}{l}\text { 1.On-board unit } \\
\text { 2.Wireless network cards } \\
\text { 3.GPS } \\
\text { 4.On-board diagnostic }\end{array}$ & $\begin{array}{l}1 \text { Guaranteeing the safety of people. } \\
2 \text { Verifying the reliability of the communication system }\end{array}$ \\
\hline $\begin{array}{l}\text { Sungwon Lee } \\
\text { (2015) }\end{array}$ & Korea & Wireless Personal communication & $\begin{array}{l}\text { 1.smartphone } \\
\text { 2.Wi-Fi communication module }\end{array}$ & $\begin{array}{l}\text { Inorer to provide energy efficient V2Pcommunication } \\
\text { and reduce the collision between safety messages. }\end{array}$ \\
\hline $\begin{array}{l}\text { Dahlia Sam } \\
\quad(2015)\end{array}$ & India & $\begin{array}{l}2015 \text { International Conference on Innovation in } \\
\text { Computing Technologies }\end{array}$ & $\begin{array}{l}\text { 1.Hybrid VANET System (vehicle } \\
\text { nodes and Road Side Unit) } \\
2 \text { Body sensors }\end{array}$ & $\begin{array}{l}\text { Giving an extra level of safety to the careless pedestrian } \\
\text { who might be hit by driver. }\end{array}$ \\
\hline $\begin{array}{l}\text { Dahlia Sam } \\
\quad(2015)\end{array}$ & India & Journal of Engineering and Applied Sciences & $\begin{array}{l}\text { 1.Vehicle nodes } \\
\text { 2.Wi-Fi module } \\
\text { 3.Road Side Unit } \\
\text { 4.Body sensor }\end{array}$ & $\begin{array}{l}\text { Reducing the road accidents and death rate of } \\
\text { pedestrian. }\end{array}$ \\
\hline $\begin{array}{l}\text { Toru Hagiwara } \\
\qquad(2015)\end{array}$ & Japan & The journal of Intelligent Transportation System & - & $\begin{array}{l}1 \text { Evaluating the performance of the pedestrian-vehicle } \\
\text { DSRC communication system. } \\
2 \text { Determining the warning position. }\end{array}$ \\
\hline
\end{tabular}


Table 2. Cont.

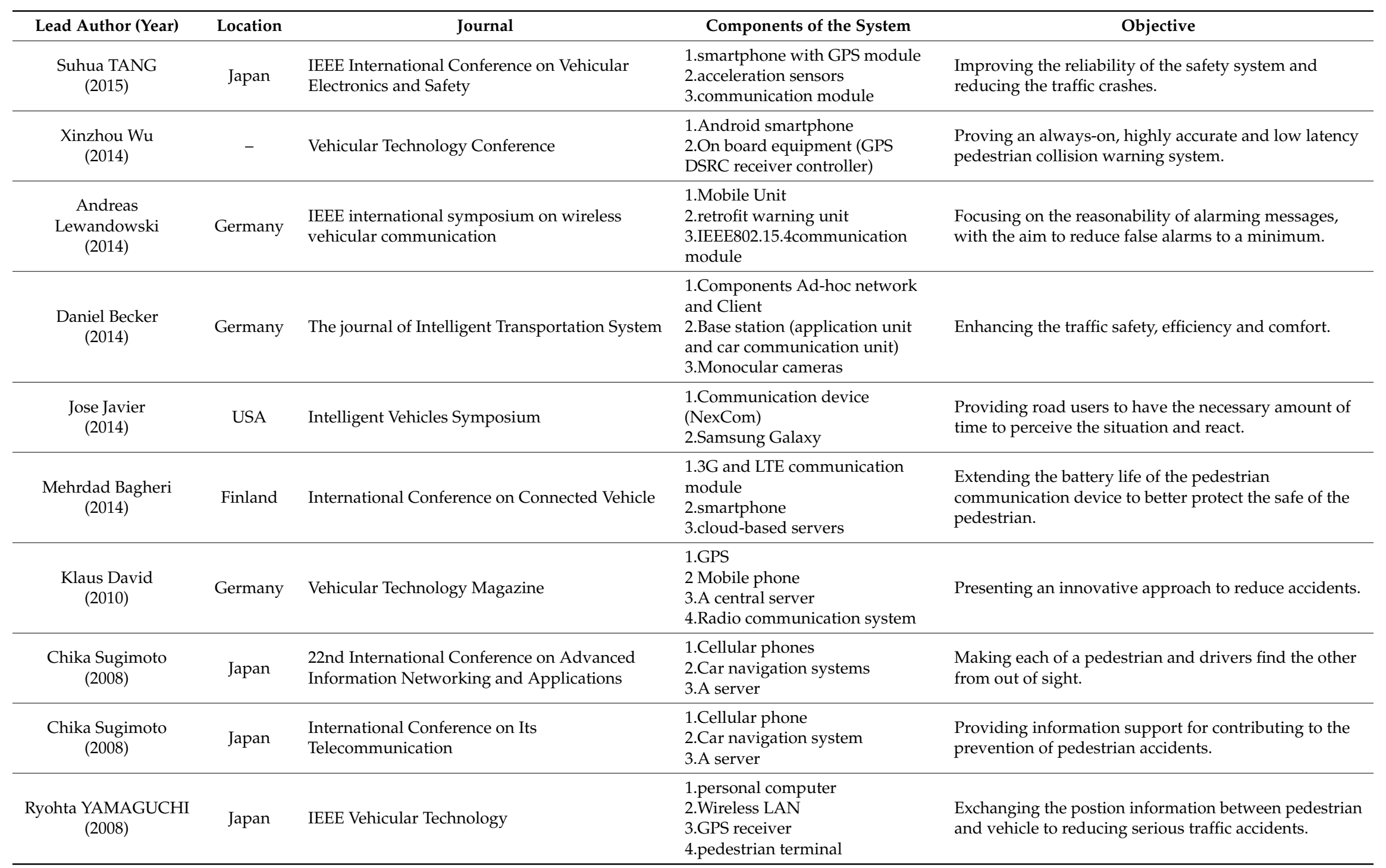




\subsection{The Factors of Influencing Data Transmission Procedure}

Car-to-pedestrian communication safety system based on the vehicular ad hoc network is a potential technology to reduce traffic accident and improve traffic safety. The safety between pedestrians and vehicles largely depends on the information exchanged each other ahead of time. Obviously, the data transmission is the core part of those systems, but the efficiency of data transmission is influenced by a large number of factors (e.g., the obstacles; the transmission distance; the packets lost rate; the state of pedestrians and vehicles). Each of the factors may trigger a fatal or unexpected consequence. Hence it is necessary to access the quality and efficiency of the communication system via accessing the efficiency of data transmission. A summary of factors influencing the data transmission is shown in Table 3.

Table 3. Factors influence the data transmission in the selected papers.

\begin{tabular}{|c|c|c|c|c|c|}
\hline \multirow[b]{2}{*}{ Lead Author (Year) } & \multicolumn{5}{|c|}{ Factors Influence the Data Transmission } \\
\hline & Obstacles & $\begin{array}{l}\text { Transmission } \\
\text { Distance }\end{array}$ & $\begin{array}{l}\text { Under Dynamic } \\
\text { Conditions }\end{array}$ & $\begin{array}{c}\text { The Transmission } \\
\text { Delay }\end{array}$ & $\begin{array}{l}\text { Packets Lost } \\
\text { Rate }\end{array}$ \\
\hline Hassan Artail (2017) & $\sqrt{ }$ & $\sqrt{ }$ & & & \\
\hline Zishan Liu (2016) & $\sqrt{ }$ & $\sqrt{ }$ & $\sqrt{ }$ & $\sqrt{ }$ & $\sqrt{ }$ \\
\hline Merdrignac (2016) & $\sqrt{ }$ & $\sqrt{ }$ & $\sqrt{ }$ & & \\
\hline Toru Hagiwara (2015) & & $\sqrt{ }$ & & & $\sqrt{ }$ \\
\hline Liu zhenyu (2015) & $\sqrt{ }$ & $\sqrt{ }$ & $\sqrt{ }$ & $\sqrt{ }$ & $\sqrt{ }$ \\
\hline Sungwon Lee (2015) & & $\sqrt{ }$ & & & $\sqrt{ }$ \\
\hline Suhua TANG (2015) & & & $\sqrt{ }$ & $\sqrt{ }$ & \\
\hline Xinzhou Wu (2014) & $\sqrt{ }$ & $\sqrt{ }$ & $\sqrt{ }$ & $\sqrt{ }$ & \\
\hline Lewandowski (2014) & $\sqrt{ }$ & $\sqrt{ }$ & & & \\
\hline Jose Javier (2014) & $\sqrt{ }$ & $\sqrt{ }$ & $\sqrt{ }$ & $\sqrt{ }$ & $\sqrt{ }$ \\
\hline Klaus David (2010) & & $\sqrt{ }$ & & & \\
\hline Chika Sugimoto (2008) & & $\sqrt{ }$ & $\sqrt{ }$ & $\sqrt{ }$ & \\
\hline
\end{tabular}

With the increasing popularity of the vehicles and extensive demand, traffic accident is one of the most severe problems faced by human. Numerous efforts are devoted by researchers and scholars to reduce and alleviate the traffic crashes. Liu et al. [42] designed an efficient V2X communication system to guarantee the safety of the pedestrians. Experiments are conducted to evaluate the performance of IEEE 802.11p for vehicle-to-vehicle communication and the Wi-Fi for the vehicle-to-pedestrian communication in terms of packets delivery rate and transmission delay under static and dynamic conditions. For closing to the real world to measure the packets delivery rate and transmission delay, the author choosing distance from $0 \mathrm{~m}$ to $400 \mathrm{~m}$ with $50 \mathrm{~m}$ interval, during the experiment, there are few obstacles and numerous obstacles on the road to investigate the factors to influence the data transmission. The results showed that the packets delivery rate of the IEEE 802.11p and Wi-Fi decrease with the transmission distance increasing. Moreover, the obstacles have a huge effect on the data transmission and the speed do not have an obvious influence on the data transmission in low-speed situations. Ultimately, it was proved that this communication safety system provided a reliable option for the safety between pedestrians and vehicles. Nevertheless, in this paper, the author just using the GPS to positioning for the pedestrians and vehicles, but he never mentioned the accuracy and preciseness of the positioning device. Accurate positioning is a prerequisite for avoiding the collision between pedestrians and vehicles, which is a potential threat to the safety of pedestrians.

Similar to the above system, Javier et al. [38] introduce a pedestrian-to-vehicle communication system based on the Wi-Fi technology. In this paper, authors investigated the GPS accuracy and the packets lost rate and transmission delay of the communication system under dynamic conditions. Two scenarios are built to validate transmission efficiency influenced by obstacles. It is proved that the obstacles have a significant impact on the signal transmission. If the signal is blocked by the obstacles, the communication distance would be significantly shorter. Although the author considered the factors of data transmission comprehensively, the communication connection between pedestrians 
and vehicles via Wi-Fi is unstable, sometimes even broken, especially in suburban. On the other hand, the GPS error is about $10 \mathrm{~m}$, which is not satisfied with the safety application and influence the reliability of the communication safety system.

Another scholar using Wi-Fi technology to design a collaborative vehicle-to-pedestrian communication safety system to take evasive action and prevent a traffic collision by Wu et al. [39]. Experiments are conducted when the pedestrian and vehicle run at different speed and different distance to evaluate the performance of the system, then recording the packets lost rate. Of course, during the experiment process, the obstacles are considered to access the system. Furthermore, the author calculated the difference between the GPS fixes and the landmarks on Google earth to measure the GPS error and proposed a collision detection algorithm to deal with uncertainty of pedestrian location and trajectory. Last but not the least, the author developed an algorithm to identify the classification of the target and false alarm for pedestrian-to-vehicle scenarios. Unfortunately, this paper has not considered the other factors which influence the data transmission to access the reliability of the communication safety system. Furthermore, this paper has not proposed an efficient solution to solve the accuracy of the positioning.

Considerable car-to-pedestrian communication safety systems are based on the Wi-Fi technology. Merdrignac et al. [45] introduced a new cooperative system based on both perception and V2P communication to protect vulnerable road users. In this paper, the author focused on evaluating perception and communication fusion. Eleven field experiments were conducted to test the proposed system containing two possible conditions: (1) no obstacles between the vehicles and pedestrian (LOS); (2) pedestrian stands behind a parked vehicle (NLOS). The results showed that the cooperative vulnerable road user protection system can benefit of the redundancy coming from the perception and communication technologies both line-of-sight (LOS) and none-LOS conditions. However, distance and obstacles between pedestrian and vehicles have a huge effect on the fusion of perception and V2Pcommunication. In the future, more efforts should be contributed to validate the transmission efficiency and the accuracy and precision of the positioning device.

Considering some limitations of the Wi-Fi technology, Hagiwara et al. [43] considered the spatial position of both pedestrians and on-coming vehicles and proposed a pedestrian-to-vehicle communication system based on the DSRC technique. The main aim of the paper was to investigate the warning position accepted by pedestrians under a dynamic environment. Field experiments were designed to validate the performance of the pedestrian-to-vehicle communication safety system. The results of the paper showed that the packets arrival rates (mostly over the $80 \%$ ) performed well when the distance between vehicles and pedestrians was $50 \mathrm{~m}$ or more. The pedestrian preferred warning position ranged from $6 \mathrm{~m}$ to $9 \mathrm{~m}$ before the conflict point. However, in this paper, the author just considered two influenced factors (the distance and packets lost rate) to validate the performance of the DSRC based communication system. The additional influenced factors are omitted, therefore, it is necessary to conduct more experiments to validate the other factors how to influence the data transmission.

David et al. [24] presented an innovative car-to-pedestrian communication safety system concept which is based on detection, filtering supported by personal profiles and context awareness, communication and warning. The author discussed the architectures of some communication safety system consisting of ad hoc/cellular network for communication to reduce the traffic accident between vehicles and pedestrians. In this study, the author conducted the experiment to validate the transmission delay. It is shown that the LET have a better low transmission delay. Then, the author proposed a hybrid solution which enable choose the most appropriate way of communication and processing setup based on the usage, availability, and context is the most promising architecture. However, author just analyzed some ad hoc/cellular architectures and proposed a concept of communication safety system, and a large number of factors influenced the data transmission are non-involved, which is the future work of the communication safety system. 
A system was proposed that relies on cellular communication and vehicular communication to detect probable vehicle to pedestrian crashes by Artail et al. [46]. This system was generally based on enabling the Road Side Unit to obtain vectors of positions of cars and nearby pedestrian, and predicted probable collision events, and accordingly informed the concerned vehicles so they can take actions to avoid traffic crashes. The reliability of this system was validated using the Network Simulator ns 2 and SUMO. The result showed that the packets delivery rate was influenced by the obstacles and a strong dependence on the separation distance between vehicle and pedestrian, where in order to achieve $80 \%$ of packets delivery rate, the distance needs to be smaller than $100 \mathrm{~m}$ without any obstacles. However, considerable assumptions in the process of simulation disturb the result of simulation, which cannot provide real data for further analysis. More field experiments are needed to validate the reliability of the communication system.

Lewandowski et al. [47] proposed a low power pedestrian protection system based on IEEE802.15.4, the performance indicators focused on the reasonability of alarming messages, with the aim to reduce false alarms to a minimum for driver to reduce their workload. Simulation ((OMNeT++ (Budapest, Hungary) and SUMO (Berlin, Germany) and field experiments were conducted to verify the reasonability of alarming messages under the conditions where the behavior different densities of pedestrian is discussed. The results demonstrated that the probability for warnings is about $36 \%$ for a distance of up to $120 \mathrm{~m}$, and the probability for warnings is around $50 \%$ for a distance smaller than $80 \mathrm{~m}$ under the scenario of one pedestrian. When the number of pedestrian tags is enlarged to 10 , the meaningful warnings probability enlarges significantly due to higher density of pedestrians, the warning probability approach to the $100 \%$ upper bound for lower distance with a lower distance.

Similarly, TANG et al. [48] presented a pedestrian-to-vehicle communication system aiming to differentiating pedestrian transmission intervals and channel access priorities so that messages from pedestrians in high risk can be quickly and reliably sent to potentially colliding vehicles. Twenty times experiments were run to evaluate the reliability of the communication system by the packets lost rate and transmission delay. The results showed that if the average packets delivery rate was less than or equal to $10^{-4}$, the percentage of pedestrians that can transmit at the shortest interval $100 \mathrm{~ms}$ should be more than $30 \%$. It is clear that the proposed system can greatly improve packets delivery rate of pedestrian in high risk. But unfortunately, the channel congestion is still a challenge which influence the reliability of the communication system. Furthermore, improving pedestrian and vehicle positioning accuracy should be taken into account in the future.

Different communication technologies have their features and advantages, Liu et al. [49] compared the performance of the Dedicated Short Range Communication (DSRC), Generation-Long Term Evaluation (LET) and Wi-Fi based vehicular system which was to sustain the V2P communication and provide a safe situation for the pedestrian. Experiments were carried out to validate the performance of the system under different scenarios, including campus, urban street, and suburban area. Packets lost rate and transmission delay in each scenario were analyzed based on the dynamic environment with none-line-of sight (numerous obstacles) or line-of-light (few obstacles). Furthermore, experiments were employed to validate the efficiency of the communication system under different distance between vehicle and pedestrian. On the basis of experiments and data analysis, the communication system based on LET is better than DSRC in terms of packets lost rate, but a larger communication delay existing than DSRC. On the other hand, the distance of the pedestrian and vehicle have a negative effect on the reliability of the communication system. Comparison of the DSRC/LET, it was proved that the Wi-Fi based communication performed well and provided a reliable communication environment for the pedestrian and vehicle. However, it is a pity that the authors have not considered the accuracy of the positioning device. Pedestrians and vehicles are moving every moment, the accurate position is an important part to avoid the traffic accident, which may have an effect on the reliability of the communication safety system.

A system considering the accuracy of location information was designed named pedestrian-to-vehicle communication system using a cellular phone and wireless communication 
technology by Sugimoto et al. [41] for improving the safety of the pedestrian. Experiments were conducted to access the performance of the communication safety system via the time for connecting, the transmission delay, and the communication distance. It was shown that the communication delay is about $20 \mathrm{~ms}$ regardless of the distance of pedestrian and vehicle. However, this paper just mentioned the accuracy of the GPS, the authors have not solved the problem. It is a difficult to show the precise location of pedestrians, because of the low location accuracy of GPS measurement. Furthermore, the other influenced factors are not evaluated. There are large rooms to improve about the system.

Not all the vehicle-to-pedestrian communication safety systems used the data transmission and accuracy of position as the judgment criteria to access the reliability of the system. Dahlia Sam et al. $[29,50]$ proposed a communication safety system which used a Hybrid VANET based on drivers' alert system to judge the reliability of the system by comparing the maximum safe speed of the normal conditions and the situation using the communication safety system. A better chance for the driver's action to avoid an accident via an alert given to the driver ahead of time. This system is developed by fusing a pedestrian body unit and the vehicles nodes in the VANET. The information is attained by the vehicular nodes and is sent by the pedestrian body unit as the input to the alert system. Under certain conditions, the simulation was employed to compare the safe speed. The results showed that the pedestrians were at risk when the vehicles' speed was only $11 \mathrm{~km} / \mathrm{h}$ without using the communication safety system. On the other hand, the pedestrians were in danger when the vehicles' speed was above $36 \mathrm{~km} / \mathrm{h}$ via the communication safety system. It was proved that the accident can be reduced to a great extent. It is acknowledged that almost all of the avoiding collision process of the communication safety system mentioned above are depended on the reaction of the drivers and pedestrians. Most of the studies assumed that the attention of the pedestrians and drivers was concentrated on to access the reliability of the communication safety system under the experimental condition and the simulated environment. But in the real world, a lot of factors influence the reaction of the pedestrians and drivers. If they have any distraction during the warning information, which maybe cause a fatal and unexpected consequence.

Therefore, a novel vehicle control system combining Hybrid VANET which use a machine intervention as an effective alternative to replace the reaction of drivers was proposed by Sam, D. et al. [1]. This system was developed integrating the hybrid VANET and the pedestrian body unit. The signals and information were exchanged between the vehicles and pedestrians through the VANET. The information and signals were sent to the vehicle control system to check the chance of an accident occurring. If the probability of an accident is high, the vehicle control system will send a signal to the automated braking system to choose an appropriate response like braking, reducing speed or changing the lane. The simulation and experiments were conducted and the result showed that the road accident can be reduced to a great extend with using the vehicle control system. Well, the simulation and experiment were employed under the limited assumptions and controlled conditions. The real traffic conditions have more factors need to be considered. More experiments can be done to explore the other factors how to affect the reliability of communication safety system.

With the development of the society, autonomous vehicle technology recently has attracted more and more researchers' attention. Hussein et al. [51] presented a collision prediction algorithm based on the autonomous vehicle and pedestrian communication, this system relay on the proposed application broadcasting the device's position to the vehicles nearby, and reciprocally, the vehicles nearby broadcasting their position to the device, thereby, building on vehicle-to-pedestrian communication. Several experiments were performed to evaluate smartphone performance and potential limitations in terms of accuracy of the collision prediction algorithms. The results showed a great performance and high detection rate to protect the pedestrians, wonderful viability of the application for vulnerable road user protection, and the advantage for the driver in the vehicle as the collision prediction algorithm detecting an approaching vehicle in advance. However, an unfavorable feature of the application is its poisoning device precise. Future works will enhance the localization of both pedestrian and vehicles. 
The battery life of the pedestrian communication device is always a challenge and obstacle for the researchers. In order to break through the barrier, Ryohta et al. [52] provided a Cooperative Reflect Transmission Scheme which is transmission control protocol using road infrastructure. This system offers real-time communications between vehicle and pedestrian terminals, and enable to save both the transmission capacity and the power consumption of pedestrian terminals. As the experimental results, in cooperative Reflect Transmission Scheme, the communication system can reduce the redundant transmission considerably and alleviate stress and workload of the driver. On the other hand, the vehicle can recognize the existence of the pedestrian earlier than in the case of depending on the pedestrian eyesight and reacting time.

Similar to the above system, in order to overcome the bottleneck of the limited battery life of smartphone, Bagheri et al. [53] proposed a method which enables development of V2P road safety applications by wireless communication and only using the existing infrastructure and device. The authors employ an adaptive multi-level approach which operates in an energy-saving mode in risk-free situations but switches to normal mode as it detects a risky situation. Considerable field experiments are carried out to verify the performance of the communication system, it is concluded that the adaptive method tackles battery life problem and makes the road-safety system practical. In the future, in order to get a better understanding of system characteristics, its communication latency and collision avoidance accuracy should be considered to improve.

Compared to the above systems, Lee et al. [54] introduced a V2P communication system based on Wi-Fi technology, which proposed energy efficient methods to enable the V2P communication and extend the battery life of handheld devices. In this paper, the author introduced a P2P GO election method, P2P clients elect a new P2P GO based on both of residential energy and number of neighbor vehicles. In order to evaluate the performance of the proposed system, the author used SUMO (sumo-src-0.29.0) and NS-2(version2, Padova, Italy) simulator. The average delivery ratio of safety message for our proposed system was investigated, it is shown that as the vehicle's speed increase, the proposed method has a lower average delivery ratio of safety messages since congestions among safety information transmitted from the device increase. On the other hand, the average amount of residual energy was also evaluated after a period time. The results presented that in the proposed method, the P2P clients are allowed to sleep in some intervals to save the energy and extend the battery life of the devices. In the future works, more experiments should be focused on evaluating the reliability of the communication system.

\subsection{The Accuracy of Positioning}

Most of the car-to-pedestrian communication safety systems judging the collision risk are based on the location of the pedestrians and vehicles. The accuracy of positioning is a prerequisite for improving the safety of the pedestrians. Therefore, it is indispensable to identify the location of pedestrians and vehicles on the road in real time to judge the collision risk and recognize their situation. The location accuracy of a car navigation system is high due to its correction technology based on the map matching and dead reckoning. But on the other hand, the location of portable GPS of the pedestrian is not high, which will may cause fatal crashes and influence the reliability of the communication safety system. There are four papers in the selected papers to analyze the accuracy of positioning as showed in Table 4, and the rest of papers just assuming that the accuracy of the location is satisfied with the requirement of the communication safety system.

Field experiment was conducted by Chika Sugimoto et al. [41] to measure the location accuracy of cellular phone GPS. The results displayed that the accuracy of the location data was $1-6 \mathrm{~m}$ in the road outdoor and was 1-3 $\mathrm{m}$ at the residential areas. The location accuracy of the GPS is not satisfied with the requirement of communication safety system, even though $2 \mathrm{~m}$ data error may cause a fatal traffic accident. Therefore, the sensitivity of the GPS module should be increased, but unfortunately, in this paper, the author just validated the accuracy of the location, an effective solution is not proposed. 
Table 4. Analyzing the accuracy of location.

\begin{tabular}{cccc}
\hline Lead Author (Year) & Journal & $\begin{array}{c}\text { Positioning } \\
\text { Device }\end{array}$ & $\begin{array}{c}\text { Location } \\
\text { Accuracy }\end{array}$ \\
\hline Chika sugimoto (2008) & $\begin{array}{c}\text { 22nd International Conference on Advanced } \\
\text { Information Networking and Applications }\end{array}$ & Portable GPS & $10 \mathrm{~m}$ \\
\hline Javier (2014) & IEEE Intelligent Vehicles Symposium & $\begin{array}{c}\text { GPS } \\
\text { RTK GPS }\end{array}$ & $10 \mathrm{~m}$ \\
\hline Xinzhou Wu (2014) & Vehicular Technology Conference & GPS & $3 \mathrm{~m}$ \\
\hline Toru Hagiwara (2015) & The journal of Intelligent Transportation System & $\begin{array}{c}\text { GPS } \\
\text { Gyrocompass }\end{array}$ & $3 \mathrm{~mm}$ \\
\hline
\end{tabular}

Another scholar Javier et al. [38] took the accuracy of the GPS into account when he designed the communication safety system. For exploring the performance of the GPS and eliminating the interference of the environmental factor, the author chose a sunny day and collected the position data attained by the GPS receiver, and compared the collected data with that of a reference station collected by Real Time Kinematic (RTK) GPS, which provided centimeter level accuracy. The results shown that the GPS error is approximately $10 \mathrm{~m}$ and the error of lateral direction is larger than that of the longitudinal direction, which indicated that the GPS is not generally satisfactory for the communication safety system even in a good condition. However, the author also have not proposed an effective solution to solve the fatal issue.

The same as the study of Chika Sugimoto, Wu et al. [39] conducted the experiment to explore the performance and the preciseness of GPS. The result showed that GPS error is calculated via comparing the difference between GPS positioning and the landmarks on the Google Map. The error is within $3 \mathrm{~m}$. In order to cope with the accurate location of pedestrians, the author proposed a collision detection algorithm embedded into the probabilistic model for reducing the collision risk of pedestrian.

Compared with the above positioning device, Toru Hagiwara et al. [43] introduced a more advanced and accurate positioning system, which is consisted of GPS, an accelerometer, and a gyrocompass. The accuracy of the system is $3 \mathrm{~mm}$ for each longitudinal and lateral positions. It is a good option for the communication safety system to recognize the location of the pedestrians and vehicles before collision.

\section{Quality of Reviewed Studies}

As we can know from Table 5 that most of the papers access reliability of the communication safety system via the quality of data transmission. In the selected eligible 20 papers, accounting for $35.0 \%$ papers validate the data transmission delay; $25.0 \%$ papers identified the relationship between packets lost rate and data transmission; $55.0 \%$ papers considered the transmission distance between vehicles and pedestrians; $30.0 \%$ papers verified the obstacles how to influence the data transmission; and $50.0 \%$ papers took the speed of pedestrians and speed of vehicles into account to explore the relationship between dynamic situation and data transmission. Nevertheless, none of papers focused on real data to do some research to access the validation quality. Furthermore, only $30.0 \%$ papers employed simulation to simulate the sample virtual situation, the complex and complicated real environment have not been taken into account. A good news is that most papers employed the experiments to verify and validate the quality of the data transmission. As for the accuracy of the positioning, it is a pity that only $20.0 \%$ papers considering the important factors during the experiment. Unfortunately, none of papers proposed a reliable solution to solve this problem. Therefore, there are much efforts should devote to improve and explore the traffic safety between vehicles and pedestrians. 
Table 5. Distribution of quality characteristics across review papers.

\begin{tabular}{ccccc}
\hline Accessing Items & Description & Score & Number of the Works & Percentage \\
\hline \multirow{2}{*}{ The packets lost rate } & Data Transmission Quality & & \\
& Include & 1 & 5 & $25.0 \%$ \\
& Not include & 0 & 15 & $75.0 \%$ \\
\hline \multirow{2}{*}{ The distance of transmission } & Include & 1 & 11 & $55.0 \%$ \\
& Not include & 0 & 9 & $45.0 \%$ \\
\hline \multirow{2}{*}{ Obstacles } & Include & 1 & 6 & $30.0 \%$ \\
& Not include & 0 & 14 & $70.0 \%$ \\
\hline \multirow{2}{*}{ Under dynamic situations } & Include & 1 & 10 & $50.0 \%$ \\
& Not include & 0 & 10 & $50.0 \%$ \\
\hline \multirow{2}{*}{ data } & Accessing the Validation Quality & & \\
\hline \multirow{2}{*}{ Simulation } & Real data & 2 & 0 & $0 \%$ \\
& Experimental data & 1 & 20 & $100 \%$ \\
\hline \multirow{2}{*}{ Experiment } & Include & 1 & 6 & $30.0 \%$ \\
& Not include & 0 & 14 & $70.0 \%$ \\
\hline \multirow{2}{*}{ The accuracy of position device } & Include & 1 & 15 & $75.0 \%$ \\
& Not include & 0 & 5 & $25.0 \%$ \\
\hline & Accessing the Accuracy of Positioning & & \\
\hline & Include & 2 & 4 & $20.0 \%$ \\
& Not include & 1 & 16 & $80.0 \%$ \\
\hline
\end{tabular}

\section{Limitations and Strengths}

The limitations of this review should be taken into consideration while interpreting the present results. Initially, the selected eligible and valid papers must be published in English, which ignore relevant literature published in other languages. Secondly, the included works focused only on the pedestrian safety, the other road users were excluded such as cyclists, bikers and other vulnerable road users. Thirdly, when we selected the papers which employed the communication technology about the vehicular networks to avoid the traffic accidents, and papers that used other techniques to avert the collision were eliminated. This review have some strengths: (1) the eligible and valid papers were extracted from the five database using an extensive search strategy and rigorously screened and picked out by predefined inclusion/exclusion criteria; (2) the quality of selected papers was valuated based on a standardized and reproducible measurement.

\section{Discussion}

Car-to-pedestrian communication safety system based on the vehicular ad hoc network is an emerging and potential technology to reduce and decrease the traffic crashes. Comparing the traditional approaches (using RADARs, LASER scanners, IR sensors and imaging sensors computer vision) to warn the drivers of some obstacles detected, there are many advantages of the communication safety system in terms of the promptness, detection distance and the ability to bypass obstacles. On the other hand, from the macroscopic perspective, the communication safety system between vehicles and pedestrian can reduce the traffic crashes and improve the traffic safety to some degree. In briefly, this safety system not only improves the safety of vulnerable road users, but also enhance the efficiency of the traffic flow.

In this review, although a mass of car-to-pedestrian communication safety systems based on the vehicular ad hoc network have been designed and validated under different traffic conditions. Different communication systems employ various communication devices to exchange the information between pedestrians and vehicles. During the research process, there are still plenty of research obstacles and questions, such as the following:

- Car-to-pedestrian communication safety system based on vehicular ad hoc network relies on exchanging information between pedestrians and vehicles. The accurate and precise information 
(position, direction, motion type) of the pedestrians and vehicles is the prerequisite to avoid crashes among vehicles and pedestrians. Due to the unstable network connections among the communication devices and the fast changing topology of VANETs. Existing communication systems using different communication devices could not provide perfect and unmistakable information each other. Furthermore, in the complex traffic situation, the influencing factors (the packets lost, the obstacles on the transmission path, the speed of the vehicles and pedestrians, and the transmission distance) will have an influence on the reliability of the communication system. Therefore, a novel ideal to make the proposed communication safety system more reliable is needed.

- It is indispensable to measure the precise location of pedestrians and vehicles in real and complicated traffic situation in order to recognize their position and judge the collision risk for car-to-pedestrian communication safety system based on the vehicular ad hoc network. In this review, there are no articles give the details how to get to the exact position of the pedestrians and vehicles. Furthermore, the accurate positioning device might be complex, expensive and huge volume which is unfit for the handheld device. It is essential to highly enhance the robustness and precision of the positioning device.

- The existing researches mainly focus on employing the experiments under certain specific conditions to validate the reliability of the communication system, which can not present the complex and complicated real world and would not get the real world experimental data to further analysis as well as improve the communication system. In addition, there is no simulation part in most papers about these communication systems, employing real data to conduct the simulation which can assess different simple or complicated or innovative solutions before implementation. It is necessary to develop a novel simulation platform to validate the car-to-pedestrian communication safety system based on the vehicular ad hoc network.

- It is acknowledged that majority of the avoiding collision process of the communication safety system mentioned above are depended on the reaction of drivers and pedestrians. Sometimes, drivers may be distracted and interfered by trivial matters and ignore the information exchanged by the communication safety system. Deceleration and baking measures have not been taken, which may cause a fatal and unexpected consequence. Owing to the limitations in the information processing abilities in human beings. Machine intervention to replace the reaction of driver should be taken into account.

- There is no doubt that most of the field experiments only consider a pedestrian carrying a communication device and a vehicle installed communication equipment in the process of verifying the reliability of communication system. There is no other interfere information to influence the action of the driver. More field experiments should be conducted to validate the reliability of the communication system under the condition where more pedestrians carrying the communication device. The communication system can distinguish the wrong warnings and reduce the workload of the driver.

\section{Conclusions}

In this paper, we threw some light on the previous works in this research field and presented a systematical as well as comprehensive review on the car-to-pedestrian communication safety system based on the vehicular ad hoc network. In order to have a strict evaluation process, this review have provided a detailed discussion and evaluation of the car-to-pedestrian communication safety system, such as the efficiency of the data transmission from the several aspects: transmission distance, the packets lost/arrival rate, the obstacles and the motion state of the pedestrians and vehicles. Furthermore, this review also have discussed the accuracy and precision of the positioning device which is prerequisite for reliability of the safety system. Unfortunately, the researchers have not designed and developed an accurate and precise positioning device to make up for the deficiency. Last but not the least, advantages and disadvantages of the communication safety system using different communication devices have also presented in this review. 
To our limited knowledge, this work may be the first systematical review of evaluating the reliability of the communication safety system based on the vehicular ad hoc network between pedestrians and vehicles. Although communication system based on the vehicular ad hoc network is a potential solution to prevent and reduce the crashes and accidents, but these systems are developing and researching. Due to the complexity of the real traffic situation, limitation of the communication technology, and the deficiency of the positioning devices, none of the developed systems have been employed in the real traffic situation. Although most of the researchers have employed experiments to validate the reliability of the safety system, but the experiments conducted under some specific conditions, which can not present the real traffic situation. It is necessary for scholars to further research and design some efficient communication system which can protect the pedestrian, contribute to the traffic safety and improve the road traffic flow efficiency.

\section{Future Works}

Further analysis of car-to-pedestrian communication safety system based on the vehicular ad hoc network recommend that the significant research and development efforts are required to deal with the following issues.

- It is a well-known fact that most of the crashes around the globe are caused by the human error. The main reason for this human error is the limitations in the information processing abilities and the reaction time for the emergence. Most of the car-to-pedestrian communication system based on the vehicular ad hoc network are depended on the reaction of the pedestrians and vehicles. Therefore, novel ideas should be proposed by employing the machine device (activating baking, steering actuation) to replace the human thinking and reacting time. When the emergence information are exchanging through the communication device, the control center can active the braking system or steering to avoid the accident.

- In the real complex transportation system, more complicated traffic situation is faced by the driver instead of one pedestrian carrying a communication device. In the future research, more pedestrians and vehicles should be taken into account. However, this communication system will offers the driver a variety of information, excessive information increases the driver's workload and hence has a negative impact on safety. Therefore, the communication system has to be appropriately designed for offering information to drivers in place of wrong warnings.

- In order to protect the pedestrians and reduce the crashes, both pedestrian handheld devices and vehicle need to constantly and with a high frequency send updating information (their location, speed and direction). Therefore, the problem is that limited battery life of communication devices is a bottleneck in realization of these communication system $[53,55]$. The future works should focus on this aspect to improve the cruising ability of these communication safety system to provide more safety information persistently.

- The reliability of car-to-pedestrian communication safety system based on the vehicular ad hoc network is depended on exchanging location information between pedestrians and vehicles. From the safety perspective, due to the accuracy and precise of the positioning device is not satisfy the requirement of the communication safety system, it is difficult to display the correct position of the pedestrians [38,43]. Therefore, future approaches will address the location enhancement of both pedestrians and vehicles to ensure a more accurate estimation of the localization through a higher calculation frequency.

Car-to-pedestrian communication safety system based on the vehicular ad hoc network as an emerging technology have a potential to reduce traffic crashes, improve the efficiency of transportation, and alleviate traffic congestion. Several suggestions recommended in this review to provide some foundation for the future research on the communication between pedestrians and vehicles to reduce traffic crashes and further improve the reliability of the communication system. Although plenty of similar communication safety system have been proposed by scholars, it is clear that car-to-pedestrian 
communication safety system is still in its initial stage. There remain considerable obstacles and challenges to break through.

Acknowledgments: The authors would like to express their appreciation to all the members of the Integrated Transportation and Logistics Planning Institute of Jiangsu University who help to review the manuscript and offer the suggestions as well as comments.

Author Contributions: Peng Jing, Wei Huang and Long Chen together searched for valid papers from five databases and read the eligible papers carefully. Wei Huang wrote the paper. All authors have read and approved the final manuscript.

Conflicts of Interest: The authors declare no conflict of interest.

\section{References}

1. Sam, D.; Velanganni, C.; Evangelin, T.E. A vehicle control system using a time synchronized Hybrid VANET to reduce road accidents caused by human error. Veh. Commun. 2016, 6, 17-28. [CrossRef]

2. Becker, D.; Schäufele, B.; Einsiedler, J.; Sawade, O.; Radusch, I. Vehicle and pedestrian collision prevention system based on smart video surveillance and C2I communication. In Proceedings of the IEEE International Conference on Intelligent Transportation Systems, Qingdao, China, 8-11 October 2014.

3. Ferguson, R.; Xu, Z.; Green, A.; Rosenthal, K. Teens and Distraction: An In-Depth Look at Teens' Walking Behaviors. Available online: https:/ /www.safekids.org/sites/default/files/documents/ResearchReports/ skw_pedestrian_study_2013.pdf (accessed on 12 October 2017).

4. FATALITY ANALYSIS REPORTING SYSTEM (FARS) ENCYCLOPEDIA. Available online: https://wwwfars.nhtsa.dot.gov/Main/index.aspx (accessed on 12 October 2017).

5. NHTSA 2015: Advancing Safety on America's Roads. Available online: https://one.nhtsa.gov/nhtsa/ accomplishments/2015/index.html (accessed on 12 October 2017).

6. Tielert, T.; Jiang, D.; Chen, Q.; Delgrossi, L.; Hartenstein, H. Design methodology and evaluation of rate adaptation based congestion control for Vehicle Safety Communications. In Proceedings of the 2011 IEEE Vehicular Networking Conference (VNC), Amsterdam, The Netherlands, 14-16 November 2011.

7. Bhumkar, S.P.; Deotare, V.V.; Babar, R.V. Accident Avoidance and Detection on Highways. Int. J. Eng. Trends Technol. 2012, 3, 247-252.

8. Rosén, E.; Källhammer, J.-E.; Eriksson, D.; Nentwich, M.; Fredriksson, R.; Smith, K. Pedestrian injury mitigation by autonomous braking. Accid. Anal. Prev. 2010, 42, 1949-1957. [CrossRef] [PubMed]

9. Geronimo, D.; Lopez, A.M.; Sappa, A.D.; Graf, T. Survey of Pedestrian Detection for Advanced Driver Assistance Systems. IEEE Trans. Pattern Anal. Mach. Intell. 2010, 32, 1239-1258. [CrossRef] [PubMed]

10. Dollar, P.; Wojek, C.; Schiele, B.; Perona, P. Pedestrian Detection: An Evaluation of the State of the Art. IEEE Trans. Pattern Anal. Mach. Intell. 2012, 34, 743. [CrossRef] [PubMed]

11. Gidel, S.; Checchin, P.; Blanc, C.; Chateau, T.; Trassoudaine, L. Pedestrian Detection and Tracking in an Urban Environment Using a Multilayer Laser Scanner. IEEE Trans. Intell. Transp. Syst. 2010, 11, 579-588. [CrossRef]

12. Nedevschi, S.; Bota, S.; Tomiuc, C. Stereo-Based Pedestrian Detection for Collision-Avoidance Applications. IEEE Trans. Intell. Transp. Syst. 2009, 10, 380-391. [CrossRef]

13. Raßhofer, R.; Schwarz, D.; Frühauf, H. Pedestrian Protection Systems Using Cooperative Sensor Technology; Springer: Berlin/Heidelberg, Germany, 2007; pp. 135-145.

14. Gandhi, T.; Trivedi, M.M. Pedestrian Protection Systems: Issues, Survey, and Challenges. IEEE Trans. Intell. Transp. Syst. 2007, 8, 413-430. [CrossRef]

15. Labayrade, R.; Royere, C.; Aubert, D. A collision mitigation system using laser scanner and stereovision fusion and its assessment. In Proceedings of the IEEE Intelligent Vehicles Symposium, Las Vegas, NV, USA, 6-8 June 2005.

16. Kubota, S.; Okamoto, Y.; Oda, H. Safety Driving Support System Using RFID for Prevention of Pedestrian-involved Accidents. In Proceedings of the 2006 6th International Conference on ITS Telecommunications, Chengdu, China, 21-23 June 2006.

17. Fujii, H.; Seki, K.; Nakagata, N. Experimental Research on Protocol of Inter-Vehicle Communication for Vehicle Control and Driver Support. In Proceedings of the Steps Forward, Intelligent Transport Systems World Congress, Yokohama, Japan, 9-11 November 1995. 
18. Sasaki, I.; Hirayama, T.; Hatsuda, T. Vehicle information networking based on inter-vehicle communication by laser beam injection and retro-reflection techniques. In Proceedings of the Vehicle Navigation and Information Systems Conference, Yokohama, Japan, 31 August-2 September 1994.

19. Mizui, K.; Uchida, M.; Nakagawa, M. Vehicle-to-vehicle 2-way communication and ranging system using spread spectrum technique. In Proceedings of the Papers of Technical Meeting on Intelligent Transport Systems, Yokohama, Japan, 31 August-2 September 1994.

20. Sam, D.; Raj, V.C. A time synchronized hybrid vehicular Ad hoc network of roadside sensors and vehicles for safe driving. J. Comput. Sci. 2014, 10, 1617-1627. [CrossRef]

21. Valade, J.; de Vaulx, L. Vehicle to Vehicle Communications: Experimental Results and Implementation Perspectives. In Proceedings of the Steps Forward, Intelligent Transport Systems World Congress, Yokohama, Japan, 9-11 November 1995.

22. Kremer, W.; Hubner, D.; Hoff, S.; Benz, T.P.; Schafer, W. Computer-aided design and evaluation of mobile radio local area networks in RTI/IVHS environments. IEEE J. Sel. Areas Commun. 1993, 11, 406-421. [CrossRef]

23. Liu, Y.; Bi, J.; Yang, J. Research on Vehicular Ad Hoc Networks. In Proceedings of the 2009 China Control and Decision-Making Conference, Shanghai, China, 16-18 December 2009.

24. David, K.; Flach, A. CAR-2-X and Pedestrian Safety. IEEE Veh. Technol. Mag. 2010, 5, 70-76. [CrossRef]

25. Sugimoto, C.; Nakamura, Y.; Hashimoto, T. Prototype of pedestrian-to-vehicle communication system for the prevention of pedestrian accidents using both $3 \mathrm{G}$ wireless and WLAN communication. In Proceedings of the 3rd International Symposium on Wireless Pervasive Computing, Santorini, Greece, 7-9 May 2008.

26. Hui, F.; Mohapatra, P. Experimental characterization of multi-hop communications in vehicular ad hoc network. In Proceedings of the ACM International Workshop on Vehicular Ad Hoc Networks, Cologne, Germany, 2 September 2005.

27. Carmona, J.L. Cars Becoming Computers on Wheels. Caribb. Bus. 2014, 42, 4.

28. Lee, U.; Park, J.-S.; Yeh, J.; Pau, G.; Gerla, M. Code Torrent: Content Distribution Using Network Coding in VANET. In Proceedings of the International Workshop on Decentralized Resource Sharing in Mobile Computing and Networking, Los Angeles, CA, USA, 25 July 2006.

29. Sam, D.; Evangelin, E.; Raj, V.C. A Novel Idea to Improve Pedestrian Safety in Black Spots Using a Hybrid VANET of Vehicular and Body Sensors. In Proceedings of the International Conference on Innovation Information in Computing Technologies, Chennai, India, 9-20 February 2015.

30. Caird, J.K.; Johnston, K.A.; Willness, C.R.; Asbridge, M.; Steel, P. A meta-analysis of the effects of texting on driving. Accid. Anal. Prev. 2014, 71, 311. [CrossRef] [PubMed]

31. Strayer, D.L.; Drews, F.A.; Crouch, D.J. A comparison of the cell phone driver and the drunk driver. Hum. Factors 2006, 48, 381-391.

32. Schwebel, D.C.; Stavrinos, D.; Byington, K.W.; Davis, T.; O’Neal, E.E.; De Jong, D. Distraction and pedestrian safety: How talking on the phone, texting, and listening to music impact crossing the street. Accid. Anal. Prev. 2012, 45, 266-271. [CrossRef] [PubMed]

33. Stavrinos, D.; Byington, K.W.; Schwebel, D.C. Distracted walking: Cell phones increase injury risk for college pedestrians. J. Saf. Res. 2011, 42, 101-107. [CrossRef] [PubMed]

34. Hatfield, J.; Murphy, S. The effects of mobile phone use on pedestrian crossing behaviour at signalized and unsignalized intersections. Accid. Anal. Prev. 2007, 39, 197-205. [CrossRef] [PubMed]

35. Wu, X.; Subramanian, S.; Guha, R.; White, R.G.; Li, J.; Lu, K.W.; Bucceri, A.; Zhang, T. Vehicular Communications Using DSRC: Challenges, Enhancements, and Evolution. IEEE J. Sel. Areas Commun. 2013, 31, 399-408.

36. Tahmasbi-Sarvestani, A.; Kazemi, H.; Fallah, Y.P.; Naserian, M.; Lewis, A. System Architecture for Cooperative Vehicle-Pedestrian Safety Applications Using DSRC Communication; SAE Technical Paper No. 2015-01-0290; SAE International: Warrendale, PA, USA, 2015. [CrossRef]

37. Sugimoto, C.; Nakamura, Y. Provision of information support by pedestrian-to-vehicle communication system. In Proceedings of the International Conference on ITS Telecommunications, Phuket, Thailand, 24 October 2008.

38. Anaya, J.J.; Merdrignac, P.; Shagdar, O.; Nashashibi, F.; Naranjo, J.E. Vehicle to pedestrian communications for protection of vulnerable road users. In Proceedings of the Intelligent Vehicles Symposium, Dearborn, MI, USA, 8-11 June 2014. 
39. Wu, X.; Miucic, R.; Yang, S.; Al-Stouhi, S.; Misener, J.; Bai, S.; Chan, W.-H. Cars Talk to Phones: A DSRC Based Vehicle-Pedestrian Safety System. In Proceedings of the Vehicular Technology Conference, Vancouver, BC, Canada, 14-17 September 2014.

40. Vrabel, M. Preferred Reporting Items for Systematic Reviews and Meta-Analyses. Oncol. Nurs. Forum 2015, 42, 552-554. [CrossRef] [PubMed]

41. Sugimoto, C.; Nakamura, Y.; Hashimoto, T. Development of Pedestrian-to-Vehicle Communication System Prototype for Pedestrian Safety Using both Wide-Area and Direct Communication. In Proceedings of the 22nd International Conference on Advanced Information Networking and Applications, Okinawa, Japan, 25-28 March 2008; pp. 64-69.

42. Liu, Z.; Pu, L.; Zhu, K.; Zhang, L. Design and evaluation of V2X communication system for vehicle and pedestrian safety. J. China Univ. Posts Telecommun. 2015, 22, 18-26.

43. Hagiwara, T.; Uchibori, D.; Hamaoka, H.; Hirano, R.; Hamaguchi, M.; Nakabayashi, S.; Munehiro, K.; Takemoto, A.; Suzuki, K. Data Transmission Performance of DSRC (5.8 GHz) typed Pedestrian-Vehicle Communication to Avoid Conflict between Right-Turn Vehicles and Pedestrians, Part 2. Int. J. Intell. Transp. Syst. Res. 2015, 13, 1-9.

44. Kim, Y.; Kim, I.; Shim, C.Y. A taxonomy for DOS attacks in VANET. In Proceedings of the International Symposium on Communications and Information Technologies, Incheon, Korea, 24-26 September 2015.

45. Merdrignac, P.; Shagdar, O.; Nashashibi, F. Fusion of Perception and V2P Communication Systems for the Safety of Vulnerable Road Users. IEEE Trans. Intell. Transp. Syst. 2016, 18, 1740-1751. [CrossRef]

46. Artail, H.; Khalifeh, K.; Yahfoufi, M. Avoiding car-pedestrian collisions using a VANET to cellular communication framework. In Proceedings of the13th International Wireless Communications and Mobile Computing Conference (IWCMC), Valencia, Spain, 26-30 June 2017; pp. 458-465.

47. Lewandowski, A.; Bocker, S.; Koster, V.; Wietfeld, C. Design and performance analysis of an IEEE 802.15.4 V2P pedestrian protection system. In Proceedings of the IEEE 5th International Symposium on Wireless Vehicular Communications (WiVeC), Dresden, Germany, 2-3 June 2014.

48. Tang, S.; Saito, K.; Obana, S. Transmission control for reliable pedestrian-to-vehicle communication by using context of pedestrians. In Proceedings of the 2015 IEEE International Conference on Vehicular Electronics and Safety (ICVES), Yokohama, Japan, 5-7 November 2015; pp. 41-47.

49. Liu, Z.; Liu, Z.; Meng, Z.; Yang, X.; Pu, L.; Zhang, L. Implementation and performance measurement of a V2X communication system for vehicle and pedestrian safety. Int. J. Distrib. Sens. Netw. 2016, 12. [CrossRef]

50. Sam, D.; Evangelin, E.; Raj, V.C. Improving road safety for pedestrians in black spots using a hybrid vanet of vehicular sensors and pedestrian body unit. ARPN J. Eng. Appl. Sci. 2015, 10, 4639-4644.

51. Hussein, A.; García, F.; Armingol, J.M.; Olaverri-Monreal, C. P2V and V2P communication for Pedestrian warning on the basis of Autonomous Vehicles. In Proceedings of the IEEE International Conference on Intelligent Transportation Systems, Rio de Janeiro, Brazil, 1-4 November 2016.

52. Yamaguchi, R.; Ikeda, D.; Nakanishi, Y.; Wada, T.; Okada, H. A cooperative reflect transmission scheme using road infrastructure in vehicle-PEdestrian communications. In Proceedings of the IEEE 68th Vehicular Technology Conference, Calgary, BC, Canada, 21-24 September 2008; pp. 1-5.

53. Bagheri, M.; Siekkinen, M.; Nurminen, J.K. Cellular-based vehicle to pedestrian (V2P) adaptive communication for collision avoidance. In Proceedings of the International Conference on Connected Vehicles and Expo, Vienna, Austria, 3-7 November 2015.

54. Lee, S.; Kim, D. An energy efficient vehicle to pedestrian communication method for safety applications. Wirel. Pers. Commun. 2015, 86, 1845-1856. [CrossRef]

55. Ikeda, D.; Horie, M.; Yamaguchi, R.; Wada, T.; Okada, H. An effective algorithm of the relative movement between vehicles and pedestrians in VPEC. In Proceedings of the ACM International Workshop on Wireless Network Testbeds, Experimental Evaluation and Characterization, Montreal, QU, Canada, 10 September 2007.

(C) 2017 by the authors. Licensee MDPI, Basel, Switzerland. This article is an open access article distributed under the terms and conditions of the Creative Commons Attribution (CC BY) license (http:/ / creativecommons.org/licenses/by/4.0/). 